\title{
Eustache Deschamps : images de lecteurs, autoportraits et toile de fond
}

\section{Virginie Minet-Mahy}

\section{(2) OpenEdition}

10 Journals

\section{Édition électronique}

URL : http://journals.openedition.org/studifrancesi/34062

DOI : 10.4000/studifrancesi.34062

ISSN : 2421-5856

Éditeur

Rosenberg \& Sellier

\section{Édition imprimée}

Date de publication : 1 novembre 2005

Pagination : 225-239

ISSN : 0039-2944

\section{Référence électronique}

Virginie Minet-Mahy, «Eustache Deschamps : images de lecteurs, autoportraits et toile de fond », Studi Francesi [En ligne], 146 (XLIX | II) | 2005, mis en ligne le 30 novembre 2015, consulté le 19 avril 2021. URL : http://journals.openedition.org/studifrancesi/34062 ; DOI : https://doi.org/10.4000/ studifrancesi.34062

\section{(c) (i) (9)}

Studi Francesi è distribuita con Licenza Creative Commons Attribuzione - Non commerciale - Non opere derivate 4.0 Internazionale. 


\section{Eustache Deschamps: images de lecteurs, autoportraits et toile de fond}

\section{Du béros à la figure exemplaire}

L'usage des figures exemplaires est un phénomène typique de la littérature antique et médiévale et relativement peu familier du monde contemporain. Entre le héros et la figure exemplaire, il y a de grandes distances qu'il importe de pointer dès le début pour tenter de cibler ensuite quel est le héros en jeu dans certains textes qui font usage des figures exemplaires. Le héros d'une littérature médiévale engagée semble se profiler comme le lecteur lui-même et comme l'auteur, le processus de lecture visant à spiritualiser, à réformer le lecteur. Le concept corollaire d'antihéros est également heuristiquement riche pour comprendre le fonctionnement des personnages bibliques et mythologiques invoqués dans les textes. A côté des personnages illustres qui sont le lot des exemples, David, Job, Alexandre, il y a bien entendu des mauvaises figures: Absalon, Nabuchodonosor. Mais la notion d'antihéros entrouvre aussi comme perspective, la mise en évidence de phénomènes d'inversion, de parodie, souvent révélateurs des processus signifiants. On peut alors sortir du strict champ de représentation dans lequel on enferme l'interprétation des figures exemplaires comme outil d'une littérature essentiellement moralisante ou encomiastique, dans la lignée des Vies de Plutarque et de Boccace (De casibus virorum illustrium).

Il s'agira donc d'analyser le lien qui associe les figures exemplaires et le lecteur/ héros, de voir en quoi les figures exemplaires participent à la construction de l'idéal de lecteur. Dans cette perspective, le texte constitue l'élément civilisateur, le lieu d'une saisie du sujet, d'une connaissance qui lui permet d'échapper à la barbarie de l'ignorance. L'image d'un lecteur aveugle auquel échappe la lettre du texte fait pendant à la figure de celui qui méconnaît Dieu et derrière lequel se profile le tyran, l'idolâtre dont Jean-Claude Mühlethaler a bien mis en évidence les traits dans son étude sur la satire médiévale ${ }^{1}$.

Le développement croissant de galerie de héros dans les textes impose de soulever la question de l'effet voulu par l'écriture dans le recours à l'béroïsation. Le texte est un lieu de construction active du lecteur. Cette vision de la question de l'héroïsation couplée à celle du lecteur découle des observations faites dans le cadre de ma thèse de doctorat qui porte sur l'esthétique et le pouvoir de l'œuvre allégorique à

(1) J.-Cl. MüHLethaler, Fauvel au pouvoir: lire la satire médiévale, Paris, Champion, 1994
(«Nouvelle Bibliothèque du Moyen Âge», 26). 
l'époque de Charles $\mathrm{VI}^{2}$. Le corpus regroupe des textes d'auteurs engagés dans les affaires du temps, sensibilisés par la guerre civile, la guerre de Cent Ans, le Grand Schisme. Dans ce cadre, l'œuvre dévoile souvent une vocation de réformation des forces sociales, et en particulier du prince, tête de la nation. C'est donc essentiellement à lui que s'adressent les textes. Le héros-lecteur s'identifie donc au prince et le texte en un lieu d'acquisition de sagesse, de connaissance, source de vérité contre le risque de la tyrannie. Mais le héros, c'est aussi l'auteur: c'est grâce à lui que les outils de rénovation morale et spirituelle sont donnés au lecteur. A travers des autoreprésentations, notamment à travers l'usage de figures mythiques, l'auteur exprime sa vocation et tend à conquérir une légitimité. C'est Christine muée en homme et le mythe de Tirésias. C'est Guillaume de Machaut et Polyphème ou Ulysse. C'est Froissart et ses inventions mythologiques ${ }^{3}$.

Cette étude se centrera de manière plus particulière sur l'usage des figures exemplaires sous la plume d'Eustache Deschamps, sur leur implication quant à l'action sur le public et quant à l'image de soi du poète.

Avant d'aborder les textes, il convient de souligner trois aspects de la figure exemplaire qui la distingue fortement du héros.

Le fonctionnement des figures exemplaires est protéique: il dépend d'une série de facteurs qui tiennent notamment à l'ancrage dans un type générique. Le Moyen Âge bouleverse certainement les représentations des lecteurs contemporains par la variété des genres pratiqués. Le terrain d'accueil influence évidemment la portée signifiante de la technique. Or on rencontre des figures exemplaires aussi bien dans les poésies lyriques que dans de vastes textes (débats, dits). Au contraire, lorsque l'on pense «héros», on voit surgir essentiellement le roman, principalement, du moins une littérature narrative. Au sein d'un même genre médiéval on peut aussi observer de grandes disparités, en ordre de grandeur aussi: dans les poésies lyriques de Deschamps, les figures abondent, alors que Charles d'Orléans en fait rarement usage. Les pratiques témoignent des projets d'écriture variés. Mais c'est aussi les sources des exemples qui sont variées, l'histoire romaine, la Bible, l'hagiographie, la mythologie, des sources historiques ou fictives, érudites ou populaires qui ne tablent pas sur les mêmes compétences de reconnaissance et d'effets sur le public. La multiplication des terrains d'accueils (genres) et des textes sources multiplient les fonctions possibles que peuvent revêtir les figures exemplaires. L'approche de cette matière est donc plus complexe qu'on ne pourrait le croire a priori.

(2) V. Minet-Mahy, Esthétique et pouvoir de l'œuvre allégorique à l'époque de Charles VI. Imaginaires et discours, Paris, Champion, 2005 («Bibliothèque du XVe siècle», 68).

(3) Voir une série de travaux dus à J. CERQuigLinIToulet, Cadmus ou Carmenta: réflexion sur le concept d'invention à la fin du Moyen Âge, dans What is Literature? France 1100-1600, ed. by Fr. CoRnILLIAT, U. Langer, and D. Kelly, Lexington, French Forum, 1993 («The Edward C. Armstrong Monographs on medieval Literature», 7), pp. 211-230.

EADEM, Sexualité et politique: le mythe d'Actéon chez Christine de Pizan, dans Une femme de Lettres au Moyen Âge. Études autour de Christine de Pizan, éd. L. Dulac et B. Ribémont, Orléans, Paradigme, 1995, pp. 83-90.
EAdem, Polyphème ou l'antre de la voix dans le Voir Dit de Guillaume de Machaut, L'Hostellerie de Pensée. Etudes sur l'art littéraire au Moyen Âge offertes à Daniel Poirion pas ses anciens élèves, textes réunis par M. ZinK et D. BoHLER, éd. E. Hicks et M. Python, Paris, Presses de l'Université de ParisSorbonne, 1995,

Eadem, Polyphème et Prométhée. Deux voies de la «création» au XIVe siècle dans Auctor et Auctoritas. Invention et conformisme dans l'écriture médiévale. Actes du colloque de VersaillesSaint-Quentin-en-Yvelines (14-16 juin 1999), éd. M. Zimmerman, Paris, Écoles des Chartes, 2001 («Mémoires et documents de l'Écoles des Chartes »), pp. 401-410. 
En plus du problème du polymorphisme, la figure exemplaire dans le cadre d'une réflexion sur l'héroïsation pose la question du rapport privilégié du personnage au texte. La figure exemplaire a un statut d'élément étranger convoqué dans un texte.

Jacques Legrand, prédicateur de la fin du XIV ${ }^{\mathrm{e}}$ siècle définit l'usage de ces «figures» comme «allegacion» (citation) qui est «le droit parement de toute rethorique et de toute poetrie, et se puet estre nommee la souveraine couleur, car par elle tout langage se demonstre meilleur, plus souverain et plus auctentique $\gg^{4}$.

La figure exemplaire est donc une couleur de rhétorique, rhétorique qui a en vue de persuader (p. 84) et constitue une sorte de citation, en somme d'élément étranger, invité dans le texte. Elle fait apparaître une tension entre le texte englobant et le texte englobés.

En tant qu'élément de la microstructure apparaissant à titre souvent discursif, comme argument, la figure exemplaire peut paraittre relativement anecdotique par rapport à la place de choix que se taille le héros au sens plus classique. Le héros est généralement le personnage central autour duquel se construit l'œuvre. Le statut de Don Juan de Molière et de Narcisse dans le Roman de la Rose de Guillaume de Lorris n'est pas tout à fait le même. Le héros de la Rose est plutôt le narrateur, le moi en quête que le personnage ovidien. La question qui se pose dès lors, c'est la relation qui s'établit entre le héros «je» et Narcisse.

Ce n'est donc pas autour de la figure exemplaire que se construit le texte, mais le projet d'écriture, de même que la volonté d'hérö̈sation du lecteur et de l'auteur, s'alimente des ressources de la technique.

Il faut donc dépasser l'idée d'ornement du discours pour envisager la logique qui préside à l'émergence des figures exemplaires et qui active leur pouvoir.

Après cette mise au point conceptuelle sur le rapport de l'héroïsation et de la figure exemplaire, je voudrais dégager un angle d'attaque pour analyser le phénomène et faire émerger la question du héros.

\section{Le héros lecteur/le héros auteur et l'usage des figures mythologiques}

Le jeu de parodie et l'aspect subversif jettent un éclairage décapant et éclairant sur les techniques d'écriture. La désacralisation des figures présente une dimension d'antihéroïsation. Mais cet antibéroïsation a des effets constructifs. Le décalage imposé par la satire instaure un type de lecture qui fait progresser la conscience du lecteur, qui le civilise. Les réflexions de Nancy Freeman Regalado au sujet des exempla dans le Roman de la Rose sont à ce titre très suggestives. Elles posent à la fois la question de l'analogie qu'introduit l'exemple par rapport au sujet et le renforcement de l'effet par le décalage que peut entretenir l'exemple et son texte d'accueil.

Une certaine dissonance entre l'exemplum et le thème qu'il est censé éclairer peut également contribuer à augmenter la signification de l'analogie. [...] En forçant le sens de certains exemples et en augmentant la tension ambiguë entre le sujet et l'exemplum, Jean de Meung amène le lecteur à réfléchir sans cesse à la signification de l'analogie (p. 68) .

(4) Jacques Legrand, Archiloge Sophie et Livre de Bonnes Meurs, éd. par E. Beltran, Paris, Champion, 1986 («Bibliothèque du XVe siècle», 49), p. 156.

(5) La terminologie est empruntée à M. Gosman, Le Discours référentiel du Quadrilogue invectif d'Alain Chartier dans Exemplum et similitudo. Alexander the Great and other beroes as points of reference in medieval literature, ed. by W.J. AERTS and M. Gosman, Groningen, Egbert Forsten, 1988 («Medievalia Groningana», 8), pp. 159-187.

(6) N. FreEman Regalado, "Des contraires choses" La fonction poétique de la citation et des exempla dans le Roman de la Rose de Jean de Meung, «Littérature», XLI, 1981, pp. 62-81. 
Il y a fondamentalement un rapport d'étrangeté qui s'établit entre exemple et texte, l'exemple est comme une «farce», un corps étranger, rapporté qui entre en tension avec le terrain d'adoption. C'est dans la tension que le sens se construit, que le dialogue doit s'établir dans le chef du lecteur pour décoder le rapport de l'exemple au programme textuel. Au fond, plus l'exemple semble étranger au discours qui l'accueille, plus l'effort de décodage est important. Dès lors, l'exigence de la lecture fait progresser le savoir.

La satire souligne particulièrement le phénomène de la tension qui a le privilège de dévoiler le pouvoir sémantique de la figure exemplaire. Les exemples traités ici, tirés de la poésie de Deschamps, sont assez parlants. En plus de l'effet de distance obtenu par l'usage parodique, les figures que l'on va présenter impliquent fortement le recours à l'intertextualité. Celle-ci suppose un pacte avec le lecteur, celui de la reconnaissance d'un indice de dialogue nécessaire entre les niveaux de textes, entre la source et l'accueil. La prise en compte de la chaine des textes à travers laquelle transite l'exemple implique de réévaluer le sens.

En plus de prendre en compte l'intertextualité on soulignera un travail de l'horstextualité.

Non seulement la figure exemplaire entretient des relations au niveau des traditions textuelles, mais elle renvoie aussi à un système de représentation socioculturelle, à un bagage imaginaire qui incarne les valeurs attachées à un milieu culturel.

Cette piste introduit le deuxième axe qui constitue la définition du répertoire du lecteur, selon Wolfgang Iser ${ }^{7}$. Le répertoire correspond grosso modo à la notion d'horizon d'attente développée par Hans Robert Jauss. Il s'agit d'une double compétence dans le chef du lecteur: son bagage littéraire (sur base duquel il évalue le nouveau texte) et l'ensemble de ses représentations et de ses valeurs imaginaires, sociales et culturelles. L'intégration de la notion de répertoire à travers l'intertextualité et l'borstextualité permet d'impliquer une théorie de la lecture dans le cadre de l'interprétation des figures exemplaires et de mettre en évidence la rhétorique, c'est-à-dire le pouvoir d'action du texte sur le lecteur. C'est en tablant sur une reconnaissance chez le lecteur que le texte ouvre un dialogue et interroge.

Il importe dans ce cadre de constater que le recours à des figures exemplaires n'est pas le privilège de la littérature: on les retrouve autant dans des textes à valeur propagandiste (comme le Songe du Vergier ou les Ordines du sacre), dans l'iconographie et dans la mise en scène de cérémonies officielles, des joyeuses entrées 8 .

Dans le cadre d'une approche particulière de la littérature engagée, les similitudes qui associent les diverses actualisations des figures sont déterminantes pour saisir le sens. On donnera pour exemple le cas de la figure de Jason, leader des Argonautes comme emblème du nouvel ordre de la Toison d'or fondé par Philippe le Bon, puis celle de Gédéon préférée par certains membres. Les deux «héros» se trouvent exploités dans la littérature bourguignonne?.

De manière assez sensible, on peut remarquer un rapprochement du discours adressé au politique et l'usage des figures exemplaires, particulièrement d'origine mythologique. La figure exemplaire agit d'autant mieux en vue d'inscrire la figure du lecteur dans le texte si elle incorpore des actualisations imaginaires, socioculturelles.

(7) W. IsER, Der Akt des Lesens, München, Wilhelm Fink Verlag, 1976. Traduction française par E. SzNycER, L'Acte de la lecture. Théorie de l'effet esthétique, Bruxelles, Mardaga, 1985.

(8) Voir M.-R. Jung, Hercule dans la littérature française du XVIe siècle. De l'Hercule courtois à l'Hercule baroque, Genève, Droz, 1966 («Travaux d'Humanisme et de Renaissance», 79), en particu- lier, chap. III.

(9) Cl. THIRY, Mythe et politique dans quelques textes littéraires des Pays-Bas bourguignons, dans Mythe et politique. Actes du colloque de Liège, septembre 1989, Paris, Belles Lettres, 1990 («Bibliothèque de la Faculté de Philosophie et Lettres de l'Université de Liège», 257), pp. 261-272. 
Les trois pistes de réflexion (parodie, intertextualité et hors-textualité) permettent de recadrer la question des figures exemplaires dans une théorie plus globale de l'esthétique de la réception. La question serait de voir en quoi le jeu des figures exemplaires participe de l'émergence de figures de lecteur, de postures et de stratégies de lecture. L'héroïsation se situerait dès lors dans l'interaction active entre le modèle proposé par la figure exemplaire et le lecteur, non plus au sens de modèle comme reflet, miroir, guide de conduite, mais au sens scientifique du terme, comme outil heuristique de questionnement de soi, comme lieu de réflexion.

Cette interaction que l'on veut souligner entre les figures exemplaires et le lecteur/héros résulte sans aucun doute du type de littérature analysé, la littérature engagée, c'est-à-dire une littérature rédigée par des auteurs conscients de jouer un rôle public grâce à leur plume.

Par ailleurs la question du héros/lecteur implique aussi la question du héros/ auteur, dans une logique où l'émergence de la conscience du pouvoir du texte est proportionnelle à l'action que celui-ci entend jouer sur son public.

\section{Hermaphrodite, Actéon et Roboam chez Eustache Deschamps et dans son milieu culturel}

Les exemples choisis qui impliquent en particulier les poésies d'Eustache Deschamps $^{10}$ sont ancrés dans une chaîne de reprises où le sens du texte se construit véritablement dans le dialogue des intertextes. Ils témoignent de la double présence de l'intertextualité et de l'hors-textualité dans l'émergence du sens, de l'implication à la fois du lecteur et de l'auteur autour du pouvoir esthétique du texte. Dans les trois cas, l'émergence du mythe induit un travail sur l'imaginaire socioculturel, politique et littéraire. Elle construit un autoportrait de l'auteur dans sa vocation de poète engagé devant son lecteur idéal, le prince.

Hermaphrodite: amour, sexualité et débat sur la réforme de la société

La figure d'Hermaphrodite dans le Lay amoureux $(306$, t. 2) apparait de manière fugace. Ce texte au apparence courtoise reprend le schéma du Roman de la Rose (le songe, l'arrivée du narrateur dans un espace idyllique, auprès d'une fontaine où il aperçoit une assemblée autour d'Amour). Hermaphrodite est le personnage qu'Amour donne en exemple à ses sujets (comme Narcisse dans le premier Roman de la Rose et Pygmalion dans le second). Le recours à ce personnage anecdotique en soi provoque un réinvestissement du langage amoureux dans la perspective d'élaboration d'un programme de réforme adressé au pouvoir ${ }^{11}$.

Lors pour mieulx veoir le convine

Me mussay soubz une aubespine

Et vi que cilz dieux leur donna

Dame et seigneur en une eschine.

Hermofondricus le decline;

(10) Eustache Deschamps, Euvres complètes, 11 vol. publiées par le marquis Queux DE SAINTEHilaire et G. Raynaud, Paris, Didot, 1878-1903 $(\ll \mathrm{SATF} \gg)$.

(11) Pour une analyse détaillée des lais et du mythe d'Hermaphrodite chez Deschamps, voir notre article à paraître «Par l'amoureuse estincelle se peut ly mondes reformer.» Le Roman de la Rose revu par
Eustache Deschamps dans De la Rose. Sur le texte, les images et la fortune du Roman de la Rose. Actes du colloque international 10-12 avril 2003, Anvers, éd. par H. Braet et C. Bel, Leuven, Peeters. Nous ajoutons ici des précisions sur le rapport possible avec Alain de Lille et avec le Policraticus de Jean de Salisbury. 
Cilz dieux ainsis le destina;

Moitié homs et moitié femme a

Et leur dist: «Cest amour encline

A tous ii. que je vous destine

L'un et l'autre vous formera,

Et sanz lui nulz homs ne pourra

Avoir sens ne bonne dotrine,

Vaillance, honeur ne discipline,

Ne parfais jamais ne sera

En renom qui cestui n'ara:

Or gart chascuns qu'il s'i encline». (vv. 123-138)

Descendre vi celle amour digne

En un char de feu sanz courtine

Tout ardant, qui fort m'espenta

Entour ot un pou de bruine.

Ceulx qui du recevoir indigne

$\mathrm{Ne}$ furent, en eulx se bouta,

Soutivement les embrasa. (vv. 139-145, t. 2, pp. 197-198)

La virent bien qu'Amour chancelle

Se chascune n'a cuer en elle

Et chascuns pour la ramener;

Or y vueille chascune ouvrer

Et chascuns si, qu'om la rappelle,

Car par l'amoureuse estincelle

Se peut ly mondes reformer. (vv. 314-320)

Alors que la société doit fonctionner sur une relation d'amour et d'échange, la cari$\operatorname{tas}^{12}$, Hermaphrodite offre une image de repli sur soi du pouvoir et de tromperie.

La lecture du fonctionnement de la figure exemplaire nécessite une appréhension globale de l'imaginaire de Deschamps: le lien établi dans son œuvre entre la question de l'amour, de Nature et de la vision de la société comme soudée par l'amour entre gouvernant et gouverné. Elle s'éclaircit aussi dans la construction d'un programme, celui des onze lais qui doivent se lire de manière globale comme un discours d'éducation princière. La remotivation du langage courtois, la complainte amoureuse en particulier y alternent de manière subtile avec un discours anticurial et un message politique plus clair. La pierre d'angle du programme de réformation, de l'appel au prince à la clairvoyance et à l'amour véritable de son peuple, semble bien se cristalliser autour de la fontaine d'Hermaphrodite, signe d'un faux amour, métaphore de la déchéance de la société aristocratique, de la chevalerie et indice d'une nécessité de réforme.

L'intertextualité renforce la lecture interne: Le Roman de la Rose de Guillaume de Lorris engage le discours amoureux dans la réflexion sur le modèle idéal de société. Plus encore, l'articulation chez Alain de Lille entre le constat de la sodomie, incarnée dans la figure d'Hermaphrodite, la plainte de Nature sur la déchéance de l'homme et la volonté d'une rénovation de l'humanité avec l'bomo novus met bien en évidence le potentiel du mythe chez Deschamps ${ }^{13}$. On trouve également chez Jean de

(12) M. Sot, J.-P. Boudet, A. Guerreau-JalaBERT, Histoire culturelle de la France. t. 1, Le Moyen Áge, Paris, Seuil, 1997, p. 167 et 205.

(13) Dans la prologue du De Planctu Naturae, l'auteur se lamente sur la déchéance morale de l'homme, symbolisée par l'homosexualité: Vénus combat contre Vénus et affaiblit, «bestourne» les genres (aussi les genres grammaticaux): «Femina vir factus, sexus denigrat honorem,/ Ars magicae Veneris hermaphroditat eum» (Alain de Lille, 
Salisbury dans le Policraticus une mention du mythe de Salmacis et d'Hermaphrodite, figure du travestissement du sage à la cour ${ }^{14}$.

Cette intertextualité est induite en partie par la torsion importante par rapport au mythe initial. Il n'est plus question de l'aventure avec la nymphe Salmacis, mais le lai de Deschamps évoque le motif du char associé au mythe d'Hermaphrodite. Ce décalage impose une relecture forte. Faut-il y voir une inversion du char de $\mathrm{Na}$ ture, celui qu'elle envoie vers le Créateur dans le but de créer l'homme nouveau? Deschamps inverse-t-il l'image du char de la création de l'homme nouveau comme attribut de celui en raison duquel il faut restaurer l'amour? Ou une allusion au char de Phaéton, l'usurpateur de Phébus/soleil qui par son orgueil et sa tromperie a brûlé la terre en voulant conduire le soleil. Dans le livre II des Métamorphoses d'Ovide, le fils de Phébus exhorte son père à lui laisser conduire le char du soleil, en signe de sa paternité. Phaéton ne peut tenir les rênes et incendie la terre. L'Ovide moralisé a glosé le texte latin et donné pas moins de six interprétations. La glose met particulièrement en évidence, dès la traduction, l'orgueil de Phaéton, sa prétention à tenir lieu de Dieu tout-puissant ${ }^{15}$. La cinquième interprétation (914-1029) assimile plus particulièrement Phaéton à l'Antéchrist, celui qui tentera à la fin des temps de se faire passer pour le sauveur... Le texte de Deschamps entend-il à faire émerger le souvenir de la fable de Phaéton dans le prolongement de celui d'Hermaphrodite et à mettre en évidence le rôle de contrefacteur d'Amour?

La filiation d'Eustache Deschamps avec Alain de Lille et Jean de Meung permet de surcroît de souder les différentes pièces de son œuvre. La Fiction du Lion, sur le «mauvais gouvernement» fait intervenir la figure de Nature dans une plainte sur l'homme. La dé-naturation ${ }^{16}$ se constitue véritablement en motif d'expression de la dégradation de l'bomo politicus. Les hommes sont pires que les bêtes, parce qu'ils ne suivent pas leur nature, se travestissent, et causent la déchéance de la société. Hermaphrodite incarne le type même du dévoiement ontologique et donc politique, social de l'humanité.

L'intention parodique et le discours politique affleurent chez Deschamps dans un jeu de reprises et d'inversions. Le mythe et la sexualité permettent de motiver la question latente depuis Alain de Lille du modèle de société et d'humanité en jeu dans le recours aux figures exemplaires, qu'il s'agisse d'Hermaphrodite, de Narcisse, de

Liber de Planctu Naturae, PL 210, col. 431). Or, Nature a un char, composé par les sept arts libéraux. Ce char revient dans l'Anticlaudianus, autre fiction d'Alain de Lille, qui inverse le ton pessimiste de la Plainte de Nature en proposant la création d'un bomo novus pour restaurer le genre humain. De la métaphore de la licence sexuelle comme signe de la déchéance de l'humanité au projet de réforme, on retrouve la structure signifiante du projet de Deschamps en même temps qu'une communauté d'images.

(14) «Qui curialium ineptias induit et philosophi vel boni viri officium pollicetur, hermaphroditus est, qui duro vultu et hispido muliebrem deturpat venustatem et virum muliebribus polluit et inestat. Res siquidem monstruosa est philosophicus curialis; et, dum utrumque esse affectat, neutrum est, eo quod curia philosophiam excludit et ineptias curiales philosophus usquequaque non recipit. Non tamen ad omnem curiam comparatio transit sed ad illam dumtaxat quae insipientis distemperatur arbitrio.», Johannes SARISBERIENSIS, Policraticus, éd. par C. WebB, Oxford, 1909 t. I, lib. 5, 10, 21. («Celui qui endosse les sottises de la cour et offre la servitude du philosophe ou de l'homme de bien est hermaphrodite, celui qui défigure la beauté féminine pour un visage dur et grossier il souille et déshonore le caractère féminin. C'est une chose monstrueuse qu'un philosophe curial et alors qu'il cherche à être deux à la fois, il est neutre, à cause de cela, la cour exclut le philosophe et le philosophe ne reçoit pas les sottises de la cour. Cependant, la comparaison n'affecte pas toute la cour, mais seulement celle qui est mal organisée politiquement à cause de la folie du pouvoir», nous traduisons).

(15) Ovide moralisé, Poème du commencement $d u$ quatorzième siècle, publié d'après tous les manuscrits connus par C. DE BOER, t. 1, Amsterdam, Johannes Müller, 1915 («Verhandeling der Koninklijke Akademie van Wetenschappen te Amsterdam, afdeeling Letterkunde», deel 15), pp. 174-194.

(16) La licence sexuelle est de mise aussi chez les animaux qui représentent les états de la société: les races différentes s'accouplent entre elles... 
Pygmalion. La forte implication du discours politique dans le recours au mythe chez Deschamps est un phénomène marquant de son époque: on le retrouve chez Jean Gerson, notamment autour de la figure d'Hermaphrodite dans le sermon Vivat rex, prononcé en $1405^{17}$, pour la réforme du royaume. Le roi par sa vie «mystique» doit assumer le modèle christique qu'il incarne et mener à la rédemption spirituelle de sa nation. Dans le jeu d'analogie entre la Trinité et les ordres de la société, Gerson associe les trois états aux trois personnes (le Père, le Fils et le Saint-Esprit); à la chevalerie incombe le puissance, le force, comme au Père et l'anti-modèle dans ce cas est Hermaphrodite, signe d'une chevalerie déchue:

Or fault avec prudence de conseilliers que force soit et constance, es chevaliers pour executer par puissance ce qui est delibere par prudence; aultrement le roy est comme corps sans cueur et sans bras. Mais helas, delit voluptueux, souldoier mauldit de peche le vilain tirant, qui couppe la gorge en baisant et murtrit en embrassant empoisonne en abruvant, comme Joab, travaille trop ceste belle vertu, en impugnant chevalerie; car n'est si fort, si constant, si allegié, si vertueux champion que Delit ne affoiblisse et gaste se il se habandonne. C'est la fontaine Salmacis en laquelle selon la fiction des poetez chacun homme qui se baigne devient moitié femme ou feminin, Hermofoditus.

Le décryptage de la filiation textuelle, grâce à la chaîne des figures exemplaires permet de jeter un autre regard sur les diverses productions de Deschamps. Il souligne que le discours sur l'amour et la procréation fonctionnent comme lieux métaphoriques d'expression des crises du temps.

\section{Actéon: interactions entre pensée politique et représentations mythiques}

Le deuxième exemple proposé permet de voir comment un même milieu culturel, littéraire et politique actualise une figure. L'émergence et le succès d'un personnage peuvent être dus à l'appel des circonstances: une certaine forme de représentation véhiculée par lui entre en dialogue avec un discours «historique». C'est le cas de la figure d'Actéon. Son potentiel imaginaire va être fortement convoqué à l'époque de Charles VI qui reçoit pour emblème héraldique le cerf-volant ${ }^{18}$. La métamorphose du chasseur Actéon en cerf par Diane, alors que l'indiscret surprend la chaste déesse au bain, offre aux poètes une occasion d'assimilation avec le roi, d'autant qu'Actéon a reçu par ailleurs dans l'Ovide moralisé une lecture christique ${ }^{19}$ et que le roi très chrétien de la France est lui-même une figure christique. Quatre auteurs au moins intègrent la figure d'Actéon dans leur texte articulant autour d'elle un message qui implique fortement l'engagement de la politique et la figure de l'écrivain: Philippe de Mézières, Christine de Pizan, Eustache Deschamps et Jean Gerson.

(17) JeAn Gerson, Euvres complètes, vol. $7 *$, par Glorieux, Paris, Desclée de Brouwer, 1960-1974, p. 1167

(18) Colette Beaune, Costume et pouvoir en France à la fin du Moyen Âge: les devises royales vers 1400 , «Moyen Âge flamboyant: XIV"-XV siècles. Revue des Sciences Humaines», CLXXXIII, 1981, pp. 126-146.

Deschamps écrit d'ailleurs une prière de Charles VI à la Vierge où le roi est représenté comme un cerf, menacé par les chiens qui attaquent aussi les brebis (peuple). Ballade 1353. «Ly cerfs legiers, signez de .xxx. cors, / Ne puet encor esprouver sa jouvente, / Et li mastins couvoiteux, vilz et ors, / A tout ravir n'a pas la gueule lente, / Les brebiz tond et escorche et cravente, / Et le cenglier fiert de sa dent crueuse; / Deux paistres sont pour estreper mon ente: / Secourez moy, Vierge tresprecieuse! // Mais en brief temps doit venir mes confors: / Ly cerfs legiers, qui ses cornes presente, / Et puissans yert tant que par ses bons pors / Et par son hurt la vermine pulente, / Mastin, sangler, fera es corps tel fente / Que memoire yert de leur fin dolereuse; / Un seul pastour sera, tel est m'entente: / Secourez moy, Vierge tresprecieuse!». (pp. 137-138, vv. 2540).

(19) Ovide moralisé, éd. cit., t. I, livre III, vv. 604-669. 
Dans le Songe du Viel Pelerin, Philippe de Mézières, ancien conseiller de Charles V allégorise le personnage de Charles VI sous les traits de Cerf Volant. L'œuvre est une allégorie compliquée d'enseignement aux princes (Louis d'Orléans, le Blanc Faucon et Charles VI). Dans le prologue, Philippe indique que son livre doit aider Cerf Volant «Comment il se doit fort garder quant il sera bien chasse de troys chiens qui sont appellez Tyre, Myre, Bouf, qu'il ne chaye en leurs laz $»^{20}$, c'est-à-dire, qu'il se garde des péchés d'avarice, de vanité et de gourmandise. Jaqueline Cerquiglini a bien mis en évidence des échos, dans le nom des chiens, par rapport au supplice d'Actéon déchiqueté par ses propres chiens après avoir été transformé en cerf ${ }^{21}$.

La menace que met en évidence Philippe de Mézières contre la bonne conduite du prince est d'ordre moral: ce sont les péchés capitaux.

Christine de Pizan, dans le Livre de la Mutacion de fortune, travestit le mythe en reportant la culpabilité de la mésaventure d'Actéon sur Meseur, veneur attaché à Vénus, plutôt que sur Diane, déesse qu'elle a par ailleurs élue comme maîtresse, au détriment de Vénus et Junon choisies par les auteurs masculins:
A Antheon l'ont bien monstré,
Qui par ses propres chiens oultré
$\mathrm{Y}$ fu, si tost com cerfs devint,
Ce scevent des gens plus de.XX.;
Mais n'avint pas, ce m'est advis,
Par Dyane aviser ou vis,
Ains fu par Meseur et ma dame,
Qui maint lieu met a feu et flame;
Mais garde soy cellui veneur,
Qui fist, a si grant deshonneur,
Par ses cors et ses huys sonner,
Aux chiens leur seigneur malmener. (vv. 4847-4858)22

L'histoire d'Actéon est insérée dans un discours sur les Anglais qui ont forligné l'héritage de Brutus et d'Arthur en trahissant le serment de vassalité au roi de France et en attaquant ses terres. Le veneur est symboliquement à rapprocher du roi d'Angleterre qui pousse les sujets/chiens du cerf/Charles VI à le trahir ${ }^{23}$. L'enjeu du mythe chez Christine associe la figure de l'auteur et le discours politique. Christine doit concilier sa dépendance à Diane et sa fidélité au roi, le cerf blessé à l'image d'Actéon. Elle assure la cohérence de son image en transférant la culpabilité de Diane sur Fortune et sur les Anglais.

La ballade 901 (t. 5, pp. 91-92) d'Eustache Deschamps associe le discours politique et la satire du mythe dans un message anticurial. On y retrouve comme chez Christine, le mélange d'un discours public, engagé et l'émergence d'une figure du moi, mais ici dévalorisée en raison de l'ancrage dans la vie curiale:

Plus sui muez en forme merveilleuse

Qu'Yo ne fut, qui en vache mua,

(20) PhilipPe DE MÉziÈres, Le Songe du Viel Pelerin, ed. by C. W. Coopland, Cambridge, University Press, 1969 , p. 86.

(21) J. Cerquiglini-Toulet, Sexualité et politique: le mythe d'Actéon chez Christine de Pizan, op. cit., pp. 83-89.

(22) Christine de PiZAn, Le Livre de la Mutacion de Fortune, ed. S. Solente, t. II, Paris, Picard, 1959 $(\ll \mathrm{SATF} \gg)$, p. 22.
(23) On retrouve dans le Ditié de Jebanne d'Arc l'assimilation des Anglais à des chasseurs: «Si rabaissez, Anglois, voz cornes / Car jamais n'aurez beau gibier! / En France ne menez voz sornes! ». Christine de Pizan, Le Ditié de Jehanne d'Arc, ed. by A. J. KenNedy and K. VARTy, Oxford, Study of Mediaeval Languages and Literatures, 1977 («Medium Aevum Monographs», New Series, IX), strophe 39, vv. 305-307, p. 35. 
Ne qu'Antheus, en la fourest doubteuse,

Quant cerfs devint, qui d'angoisse sua

Devant ses chiens dont l'un mort le rua,

Pour la forme bestial qu'il avoit;

Mais j'ay trop pis; cheval suis, qui me voit,

Viel, recreant, dont Nature s'esmaie;

Et qui pis est, l'en m'envoie tout droit,

Comme viel roucin, mourir à la Saussaye.

Qu'est devenu ma jonesce joyeuse?

Ou est le temps que mes corps s'esprouva?

Qu'est devenu ma force vertueuse?

Ou est Amour qui lors me gouverna?

Ou est un seul de ceuls qui lors m'ama?

Chascuns me fuit; nul d'eulx ne me congnoit,

Car viellesce sanz cause me deçoipt,

D'omme en cheval pour moy muer s'essaye,

Qui me tramet desor, comment qu'il soit,

Com viel roucin, mourir a la Saussaye.

En ce lieu a mainte religieuse;

Beguinaige est que li roys y fonda;

Les dames ont de droit, et leur prieuse,

Les vieux chevaulx du roy: sont menez la

Quant usez sont; mais on leur coupera

L'oreille avant; or je ne sçay que doit;

Je suis menez auques par cest endroit;

Cheval me sens; doleur n'est que je n'aye;

Aller me fault, se Dieux ne me pourvoit,

Com viel rouncin, mourir a la Saussaye.

L'envoy

He! jeunes gens, avisez par deça:

Vieulx devendrez, il n'est chose plus vraie.

Pensez de vous; car lors l'on vous menrra,

Com viel roucin, mourir a la Saussaye.

Deschamps désacralise complètement le mythe d'Actéon en le réduisant à la dégradation de l'homme à l'état d'animal, contre Nature. La cour est un lieu de bestialité où l'homme se dénature et trahit son identité et sa parole. Les mutations d'Io en vache et d'Actéon en cerf servent de termes de comparaison pour la mutation du moi, pire, en cheval, en roncin vieux et inutile. Le cheval est un animal qui peut être attaché à la tromperie et à la stupidité, comme l'a bien montré Jean-Claude Mühlethaler à propos de Fauvel (cheval de l'apocalypse). Mais les Métamorphoses offrent aussi un cas de transformation en cheval, au livre II, celle d'Ocyrhoé. Celle-ci est la fille du centaure Chiron, mi-homme, mi-cheval (fils de Chronos qui s'était métamorphosé en cheval pour s'accoupler avec la nymphe Philyre) et de Chariclo. Douée du don de prophétie, elle révèle le destin d'Esculape et subit la punition pour avoir abusé de la divination. L'association entre le prophétisme et la figure de l'équidé, que l'on retrouve par ailleurs dans la symbolique biblique ${ }^{24}$, est particulièrement intéressante 
en relation avec la poésie et la figure de Deschamps. La posture du prophète est un des masques qu'aime adopter l'auteur engagé, notamment Deschamps pour parler au prince $^{25}$. N'y a-t-il pas allusion implicite au mythe et à la figure «prophétique» du moi, satirisée? Le poète/homme de cour au seuil de la vieillesse est transformé en animal, dégradé: il subit le même supplice qu'Ocyrhoé, celle qui a révélé le futur d'Esculape. L'Ovide moralisé a donné en ce sens une glose intéressante de la fable. Dans le Livre II, vv. 3145-3301, une première interprétation de la fable assimile Esculape au Christ («mire», sauveur de l'humanité) et Ocyrhoé à une prophétesse, une sibylle mais qui a mal usé de son don de devin ${ }^{26}$. Dans la seconde glose, elle est assimilée à Synagogue l'orgueilleuse qui malgré sa connaissance de la prophétie refuse de reconnaître le Christ comme messie annoncé.

Le don de prophétie et le détournement de la vérité couplés avec l'image du cheval consonent avec la situation de Deschamps. Contre Nature le moi à la cour devient un cheval: sa parole inspirée est détournée de sa fonction de vérité. En raison de la pression exercée par l'entourage, le poète porteur d'une parole vraie est conduit à se détourner de sa fonction et à travestir son don de prophétie.

Le mythe d'Actéon est un clin d'œil à l'animalisation du prince (le roi sans lettre est un âne couronné) et un prétexte à l'expression d'une bestialité qui touche l'homme de cour. La transformation du poète en cheval est signe de servitude, celle de la parole elle-même qui, dans le monde aveugle de la cour, ne peut assurer sa mission de vérité. On retrouve par ailleurs le mythe d'Actéon dans une ballade de plainte de la ville de Vertus, celle dont est originaire Eustache Deschamps.

\section{Ballade 845 (t. 5):}

Guerre me font tuit li iiii. element, Les dieux de l'air, de feu, d'eaue et de terre:

Mars me destruit par son embrasement

Et Saturnus par froit me vient requerre

Cerès mes blez acravante et atterre

Et mes vingnes a destruictes Bachus;

Jupiter pleut, qui de gresil m'enserre:

De tous ces maulx est servie Vertus.

Arse ay esté toute generalment

Par ceulx de Bruth, de l'ille d'Angleterre,

Puis m'a forgé foudres espessement

Vulcans li dieux; contre moy Fortune erre

Tant que ne sçay fruis, blez ne vins enquerre;

Mes noms est trop arrebours entendus,

Chascuns me fuit; qui bien en veulx en querre,

De tous ces maulx est servie Vertus.

Vertus n'est pas; on m'appelle autrement

Confusion, s'en est la droitte serre;

Fermer me vueil, maisonner ensement

(25) J.-Cl. MüHLETAHLER, Le poète et le prophète. Littérature et politique au XVe siècle, «Le moyen français», XIII, 1983, pp. 37-57. IDEM, Les masques du clerc pour parler aux puissants. Fonctions du narrateur dans la satire et la littérature «engagée» aux XIVe et XIVe siècles, «Le Moyen Âge», XCVI/2,
1990, pp. 266-286.

(26) «Ochiroé devint jument, / Qui ot esté sage devine,/ Quar le grant sens et la douctrine / Dont Dieus l'avoit enluminee, / Com fole et male endouctrinee / Aploia a maives usage. » (vv. 32043209) 
Et n'ay de quoy, ne riens ne puis acquerre:

Autre place me convandra conquerre

Et autre nom, le mien est confondus;

Deserre suis et chascuns me fait guerre:

De tous ces maulx est servie Vetus

L'Envoy

Prince, et vous dieux et deesses, de gent

Qui les fourmes muez completement

Comme jadiz fu mué Antheus

Muez mon nom et ostez mon tourment;

Biens me deffuit, Atropos m'entreprent:

De tous ces maulx est servie Vertus.

Vertus voit son nom mué, en raison de sa destruction par les Anglais. Elle est privée de ses ressources naturelles, elle a perdu son indépendance, sa «suffisance»... Comme Deschamps, perd son indépendance à la cour, devient animal. Le nom du poète, Deschamps semble d'ailleurs une création puisque Eustache se nomme en réalité Morel: le nom de Deschamps, comme sa ville «des champs», suggère une figure champêtre, celle de l'indépendance et de la liberté (notamment financière) revendiquée par le poète ${ }^{27}$. Le même malheur les touche, lui poète des champs et sa ville: la privation de liberté et l'aliénation.

On retrouve à nouveau chez Jean Gerson, dans le même sermon Vivat rex l'exploitation du mythe d'Actéon. Le ton anticurial est net, dans la lignée de celui de Deschamps: Gerson dénonce l'attitude des serviteurs qui médisent sur leur maître: «Si sont souvent les serviteurs comme les chiens Actheon qui desrompent et rongent leur propre maistre.», Vivat rex, p. 1167. Le chancelier de l'Université de Paris renoue néanmoins avec la valeur sacrée associée au cerf, alors que Deschamps en faisait un motif de bestialité. Jean Gerson joue d'ailleurs sur l'ambiguïté et l'ambivalence du cerf comme symbole du roi et symbole de l'âme. Dans La Mendicité spirituelle (317, vol. 7 , p. 245), l'âme est comparée au cerf qui recherche la fontaine de vie, mais qui est menacée par les ennemis d'enfer, les chiens:

Oroison sus ce mot: Sicut cervus, etc - O fontaine de vie, sire Dieu tout puissant, je suy la povre, lassee, dechassee, et traveillee qui a l'exemple du cerf desire venir et retourner a vous pour avoir ma recreacion et consolacion encontre la grande chaleur et la dure soif que j'ay a cause des ennemis d'enfer qui me dechassent par le desert de ce monde sans cesser, a grant nombre de chiens trez fiers, tres mordans et agus. Cilz chiens sont les diverses cures et sollicitudes mondainnes qui en maintes guises me demordent et dessirent, par quoy je suy vague, sanz repos trouver ou dit desert et ne puis maiz respirer se ie ne viens a vous qui estes fontaine de vie et lieu de refuge pour moy refroidier et seurté donner.

La pluralité des actualisations d'une même figure exemplaire sur fond d'un même symbolisme sociopolitique témoigne du dynamisme signifiant de la technique. Elle implique un jeu de reconnaissance et de décryptage complexe. Prise isolément, elle ne dépasse pas le niveau de l'anecdote, alors que dans le réseau intertextuel et socioculturel, elle agit comme outil à la fois pour pénétrer le monde imaginaire de l'auteur, sa propre figure et le pouvoir du texte.

(27) L. KENDRICK, La poésie pastorale d'Eustache Deschamps: miroir des mentalités à la fin du XIV siècle, "Romanistische Zeitschrift für Literaturgeschichte", VII, 1987, pp. 38-44. 
Roboam/Charles VI et la figure du poète vieux

Un troisième exemple permet d'apprécier encore chez Eustache Deschamps la complexité du travail de la figure exemplaire et l'interaction entre le discours engagé et l'image de soi que construit le poète.

Nombre de pièces lyriques de Deschamps expriment la douleur de la vieillesse, la décrépitude du corps devant l'avancement de l'âge. L'analyse de certaines figures exemplaires, notamment celle d'Actéon, associée à l'image du poète vieux roncin à la $\operatorname{cour}^{28}$, entrouvre la possibilité de réévaluer la dimension anecdotique, la plainte réaliste à l'égard de la vieillesse. Deschamps ne construit-il pas en partie un tableau déprécié du moi poétique qui traduit le désenchantement devant un pouvoir politique «arriviste», rejetant le conseil des anciens? On connaît la proximité de Deschamps et du parti des «Marmousets», les anciens conseillers de Charles V, ralliés au pouvoir à la majorité de Charles VI (1388). Mais, Charles VI va délaisser petit à petit les anciens conseillers.

Deschamps compare fréquemment Charles VI à Roboam, le fils et successeur de Salomon, le roi sage, qui cause la ruine de son royaume. Il assimile le malheur politique de Roboam à son éviction des vieux conseillers et à sa confiance en des jeunes inexpérimentés. C'est le message final de la Fiction de l'Aigle sur le gouvernement des princes:

Si comme du filz Salemon,

Roboan, qui a despitée

La science des vieulx donnée;

Aux jeunes crut, pour ce lison

Que sa terre fut divisée

A Jeroboan et donnée

Qui en ot la plus grant parson.

Soit donc doctrine a ce menée

Que le conseil des vieulx ne hée

En cest secle li jeunes hom,

Car leur prudence est afermée

Par grans cours de leur vie usée. (vv. 585-595, t. 6, p. 167)

La représentation du prince, élisant des conseillers jeunes, inexpérimentés et celle du poète vieillissant, désenchanté de la cour, lieu d'aliénation, consonent. En même temps que le prince est décrit comme un personnage qui n'écoute que le conseil des jeunes et délaisse l'expérience et le savoir ancien, le poète donne à voir une image de lui vieillissant et objet de dérision à la cour en raison de sa déchéance physique.

Fuiez, viellars, ne vous monstrez jamais,

Car Roboan ne vous a point en grace;

Il ne veult pas telz gens en son palays

$\mathrm{Ne}$ ensuir de son pere la trace;

Aux jeunes foulz se deduit et soulace,

Prant et ravit par le conseil d'iceulx,

Croire ne veult les prodommes ne ceulx

Que Salomon avoit mis en office;

De les oir est fel et despiteux: 
L'en het par tout droit, raison, justice

(ballade 1249, t.

6 , vv. $1-10$, p. 263$)$

Prince, vueille vous souvenir

De Roboan, qui voult tenir

Des jeunes la fole cuidance

Et des anciens departir,

Dont povre devint; sanz mentir,

Saiges se doit garder d'enfance. (ballade 1215, envoi, vv. 37-42, t. 6, p. 209)

La figure du moi vieux est mise en balance avec celle du prince dans la ballade 1253 qui raconte sous forme de fable l'histoire d'un paysan qui méprise son fidèle chien lorsqu'il devient vieux, pour lui préférer un jeune chiot:

Bien voy ceste figure et tien ;

Reduire la puis proprement

A mon service, et pour ce vien

A conclure semblablement :

Quant j'ay servi treslonguement,

Lors vient ingratitude et bruit ;

D'estat me despointe et me nuit.

Las! ma viellesce que fera?

Bien puis dire com vray instruit :

Quant fruit fault, desserte s'en va.

L'envoy

Prince, faictes faire autrement

A ceuls qui servent loyaument,

Vostre regne mieulx en vauldra ;

$\mathrm{Ne}$ faictes com le paisant

Fist a son chien mauvaisement.

Quant fruit fault, desserte s'en va. (ballade 1253, vv. 21-36, t. 6, p. 270-271)

Le rapprochement de la figure du moi et du prince, grâce notamment à la figure exemplaire permet-elle de dépasser l'anecdote de la description des syndromes physiques de la vieillesse? Deschamps écrit-il des poèmes réalistes lorsqu'il se montre en proie aux affres du temps ou associe-t-il à la dérision de soi une proclamation sur son statut précaire de poète à la cour, de voix criant dans le désert, comme celle du prophète face à un auditoire aveugle?

Ce dernier exemple permet de conclure sur les liens très subtils que le système des figures exemplaires tisse avec un réseau de représentations hors du texte et que l'auteur convoque pour provoquer une participation active du lecteur de va-et-vient entre son bagage, son répertoire et le réinvestissement littéraire. Dans le dialogue texte/lecteur que la figure exemplaire convoque se joue une réflexion sur le statut $\mathrm{du}$ lecteur. La figure exemplaire implique le lecteur dans une mise en question et éventuellement une réformation. Elle fonctionne particulièrement bien lorsque l'interlocuteur est le pouvoir, le prince en l'occurrence, qui reçoit un message de rénovation. Inévitablement, l'inscription dans le texte de la figure du lecteur par la voie particulière des personnages mythologiques ou bibliques sollicite l'auteur. Le texte se donnant vocation d'éclairer le lecteur, de le sortir de l'aveuglement, de la tyrannie, il engage aussi fortement la responsabilité de l'auteur en tant que porte-voix de la 
vérité. Derrière le réinvestissement des mythes païens se cache souvent l'expression de projets d'écriture.

Le constat de l'émergence d'une héroïsation qui agit hors du texte sur les figures d'auteurs et de lecteurs ouvre la possibilité d'investigations multiples, sur les relations qui unissent discours politique, public et figures mythiques, sur l'expression de la subjectivité de l'auteur à travers le mythe, bref sur le potentiel métapoétique du discours des figures exemplaires.

VIRGINIE MINET-MAHY 